\title{
SENESCENCE IN ORGANISMS WITH CLONAL REPRODUCTION AND COMPLEX LIFE HISTORIES
}

\author{
Maria E. Orive* \\ Department of Integrative Biology, University of California, Berkeley, California 94720
}

Submitted August 27, 1993; Revised January 21, 1994; Accepted February 25, 1994

\begin{abstract}
A population genetics model is developed predicting the fate of alleles affecting life-history attributes in organisms with complex life histories, including clonal reproduction and indeterminate growth. Such organisms are widespread and found in many ecologically important groups, including marine invertebrates such as corals and sponges, and most higher plant taxa. The evolution of senescence (here defined as a decrease in fitness components with age or stage) by the action of alleles having negatively pleiotropic stage effects is investigated in such organisms. The spread of these alleles depends on the sensitivity of the population growth rate, a measure of fitness, to changes in life-history parameters that for this model are the entries of the stage transition matrix. Examples from a published demographic study show that, for several cases examined, alleles increasing early survival or early reproduction at the cost of decreased late survival will not be favored. Clonal reproduction acts to retard the evolution of senescence, although by itself the existence of clonal reproduction in an organism does not preclude the evolution of senescence.
\end{abstract}

The evolution of senescence has occupied a central role in life-history theory because of an apparent contradiction: Why should natural selection act to decrease an organism's ability to survive? The majority of work on this question has dealt with organisms exhibiting simple age structure (see, e.g., Charlesworth 1980; Finch 1990; Rose 1991; Stearns 1992). In the theory of age-structured populations, a weakening of selection in older age-groups is expected owing to the decreased proportional contribution of these age-groups to the population gene pool (Medawar 1952; Williams 1957; Hamilton 1966; Charlesworth 1980). Two major theories describe how this weakening of selection with age might allow the evolution of a decrease in fitness components with age, that is, senescence: the first is the accumulation of deleterious mutations affecting later age classes, permitted by the decreased force of selection against them; the second involves genes having positive effects on early fitness components while having negative pleiotropic effects on later fitness components (for recent discussion, see Stearns 1992; Partridge and Barton 1993). There are also, of course, nonevolutionary theories for senescence, including the inevitable effects of cellular wear and the accumulation of toxins or other damage (Comfort 1956; Finch 1990).

Many organisms, however, do not exhibit simple age-structured life histories.

\footnotetext{
* Present address: Department of Genetics, University of Georgia, Athens, Georgia 30602.
} 
A life-history trait suggested as a mechanism allowing escape from senescence is the ability to reproduce clonally or asexually in addition to reproducing sexually. Clonal reproduction, seen often in plants and marine invertebrates, involves the formation of new individuals from somatic or germline tissues that have not undergone meiotic division. Some types of clonal reproduction include fission, budding, fragmentation, and polyembryony (Jackson et al. 1985; Hughes 1989). The number and variety of organisms that undergo clonal reproduction is enormous; nearly all higher taxa in plants and at least two-thirds of the metazoan phyla contain species with clonal reproduction (Hughes 1989; Grosberg 1992). Clonal organisms often have life histories that are better described by morphological or developmental stage classes than by age classes, and, unlike age-structured populations, individuals in a particular stage class may move to several different classes or remain in the same class (Caswell 1985, 1989). For example, mortality rates in some corals have been found to depend strongly on colony size (Hughes and Jackson 1985), and vegetatively distinct stages in plants have been shown to provide useful categories in demographic studies (Werner and Caswell 1977; Bullock 1980).

These differences can have profound effects on important aspects of the evolution of these organisms, such as effective population size and the strength of genetic drift (Orive 1993), patterns of growth and morphology (Coates and Jackson 1985; Harper 1985), and levels of selection (Buss 1985, 1987). In order to examine the role these life-history attributes may have on the evolution of senescence, it is necessary to expand the existing population genetics theory. For such organisms, fitness components are stage specific rather than age specific. Thus, senescence would be expected to take the form of a decrease in fitness components with stage.

The question of whether organisms that reproduce clonally, and thus have no clear distinction between "soma" and "germline," should evolve senescence dates back to Weismann $(1893,1904)$. Recent writing on this subject has been extensive; authors have differed, however, on the importance placed on a distinction between germline and soma (Bell 1984, 1992; Finch 1990, chap. 4; Rose 1991, chap. 5; Watkinson 1992; Partridge and Barton 1993). It seems that the important characteristic for the evolution of senescence should be whether there is a decrease in the strength of selection with age or stage. If offspring of a particular organism are in all ways identical to that organism, then there are no age-specific or stage-specific differences in the life history and thus no change in the force of selection. A single-celled organism that reproduces by binary fission, producing two identical cells, would then be expected to show no signs of senescence, when defined as a decrease in fitness attributes with age. Whether the products of fission are identical is not, however, easy to ascertain; Bell (1988) states that in Paramecium, for instance, the anterior and posterior fission products can be distinguished for an hour or two after fission.

In clonal organisms, an important distinction arises between the genetic individual, or "genet," and the physiologically distinct but genetically identical parts of a clone, the "ramets." This distinction raises the question of what constitutes an individual (Buss 1985, 1987) and thus where one should look for senescence. The 
problem is further enhanced by the use of the term clonal senescence, which is usually taken to mean a decrease in fitness in a clonal lineage seen after many generations of strictly asexual propagation (see Bell 1988). Such a decrease might be the result of the accumulation of mutations and Muller's ratchet (Muller 1964) within a clonal lineage, not of "individual" senescence as defined above. Since evolutionary theories of senescence deal with genetic causes, it might seem that the genetic individual is the level of interest. However, the physiological individual, or ramet, is the level at which life-history attributes differ. Ramets of the same genotype, if at different stages, would be expected to differ in probabilities of survival, fecundity, and so forth, and these are the attributes in which senescence will be manifested. This will be true whether the physiological individual is made up of many repeating modules, as found in a coral colony, or of a single module, as in a sea anemone. In this article, the term individual will refer to the physiological individual, and population growth will be measured in numbers of these individuals.

In this article I will first develop a population genetics model for the fate of alleles affecting life-history attributes in a diploid population with clonal and sexual reproduction. The model will be a generalization of Charlesworth's (1970, 1974, 1980) extensive work on age-structured populations, developing the theory that formalizes Caswell's $(1978,1985,1989)$ idea of the dependence of life-history evolution on eigenvalue sensitivities. Demographic data taken from a published study of clonal corals will be used to give numerical examples and allow further analysis, focusing on trade-offs between fitness components at different stages of the life history.

\section{MODEL}

For simplicity, we consider a population of $N$ diploid monoecious organisms undergoing random mating. There are $n$ demographically distinct life-history stages $(1,2, \ldots, n)$. These can be size classes or any morphological or developmental class. Stage 1 individuals are "newborn" individuals, and this stage can only be reached through sexual reproduction; individuals cannot remain in stage 1. The demography of the population is characterized by a transition matrix, $\mathbf{A}$, whose entries describe the transitions between states for individuals (Leslie 1945; Lefkovitch 1965). This matrix is assumed to be independent of time, although the theory for time-dependent transition matrices would be similar. Then

$$
\mathbf{N}_{(t)}^{\langle i, j\rangle}=\mathbf{A}^{\langle i, j\rangle} \mathbf{N}_{(t-1)}^{\langle i, j\rangle},
$$

where the $\langle i, j\rangle$ superscript indicates a population of individuals with an $A_{i} A_{j}$ genotype at some locus of interest. We let $N_{(x, t)}^{\langle i, j\rangle}$ be the number of stage $x$ individuals at time $t$, and $a_{x k}^{\langle i, j\rangle}$ be the $(x, k)$ th entry of the $\mathbf{A}^{\langle i, j\rangle}$ matrix.

In order to investigate alleles that affect life-history parameters (for our purposes, the entries of the A matrix), we need equations for changes in allele frequencies in terms of some measure of fitness. Allowing $p_{i}(x, t)$ to be the frequency of allele $A_{i}$ in individuals of stage $x$ at time $t$, and $B(1, t)$ to be the number of newborn individuals at time $t$, we find that 


$$
\begin{aligned}
p_{i}(x, t) & =\frac{\sum_{j} N_{(x, t)}^{\langle i, j\rangle}}{\sum_{i} \sum_{j} N_{(x, t)}^{\langle i, j\rangle}}, \text { for } x \neq 1, \\
p_{i}(1, t) & =\frac{\sum_{j} \sum_{x} a_{1 x}^{\langle i, j\rangle} N_{(x, t-1)}^{\langle i, j\rangle}}{B(1, t)},
\end{aligned}
$$

and

$$
B(1, t)=\sum_{i} \sum_{j} \sum_{x} a_{1 x}^{\langle i, j\rangle} N_{(x, t-1)}^{\langle i, j\rangle},
$$

for density- and frequency-independent population demographics, if we let $N_{(x, t)}^{\langle i, j\rangle}=N_{(x, t)}^{\langle j, i\rangle}$

To describe the number of individuals of a particular stage and genotype at some time $t$, we need to determine when these individuals were newborns and use the allele frequencies of newborns at that time. We define a new matrix, $\tilde{\mathbf{A}}^{\langle i, j\rangle}$, whose entries $\tilde{a}_{k l}^{\langle i, j\rangle}$ give the probability of having been in stage 1 one time unit in the past, given the organism is in stage $k$ now, and we make stage 1 an absorbing state. That is, given that the population is in a stable stage distribution with $N_{(x)}^{\langle i, j\rangle}$ as the number of stage $x$ individuals,

$$
\begin{gathered}
\tilde{a}_{k l}^{\langle i, j\rangle}=\frac{a_{k l}^{\langle i, j\rangle} N_{(l)}^{\langle i, j\rangle}}{\sum_{x} a_{k x}^{\langle i, j\rangle} N_{x}^{\langle i, j\rangle}}, \\
\tilde{a}_{11}^{\langle i, j\rangle=1} \text { and } \tilde{a}_{1 l}^{\langle i, j\rangle}=0, \text { for all } l \neq 1 .
\end{gathered}
$$

The probability that an individual currently in stage $k$ was in stage 1 , by $\tau$ time units ago, is then

$$
\left[\tilde{a}^{\langle i, j\rangle}\right]_{k 1}^{\tau},
$$

which is the $(k, 1)$ th entry of the $\tilde{\mathbf{A}}^{\langle i, j\rangle}$ matrix taken to the $\tau$ th power. The probability that the individual left stage 1 exactly $\tau$ time units ago is then $\left[\tilde{a}^{\langle i, j\rangle}\right]_{k 1}^{\tau}-$ $\left[\tilde{a}^{\langle i, j\rangle}\right]_{k 1}^{\tau-1}$. This allows us to write

$$
N_{(x, t)}^{\langle i, j\rangle}=\sum_{\tau=1}^{\infty}\left[\left(\tilde{a}^{\langle i, j\rangle}\right)_{x 1}^{\tau}-\left(\tilde{a}^{\langle i, j\rangle}\right)_{x 1}^{\tau-1}\right] p_{i}(1, t-\tau) p_{j}(1, t-\tau) B(1, t-\tau) .
$$

Inserting this expression into equations (2a) and (2c) gives

$$
\begin{aligned}
p_{i}(1, t)= & \sum_{j} \sum_{x} a_{1 x}^{\langle i, j\rangle} \sum_{\tau=1}^{\infty} p_{i}[1, t-(\tau+1)] p_{j}[1, t-(\tau+1)] \\
& \times \frac{B[1, t-(\tau+1)]}{B(1, t)}\left[\left(\tilde{a}^{\langle i, j\rangle}\right)_{x 1}^{\tau}-\left(\tilde{a}^{\langle i, j\rangle}\right)_{x 1}^{\tau-1}\right]
\end{aligned}
$$


and

$$
\begin{aligned}
B(1, t)= & \sum_{i} \sum_{j} \sum_{x} a_{1 x}^{\langle i, j\rangle} \sum_{\tau=1}^{\infty} p_{i}[1, t-(\tau+1)] p_{j}[1, t-(\tau+1)] \\
& \times B[1, t-(\tau+1)]\left[\left(\tilde{a}^{\langle i, j\rangle}\right)_{x 1}^{\tau}-\left(\tilde{a}^{\langle i, j\rangle}\right)_{x 1}^{\tau-1}\right]
\end{aligned}
$$

as the frequency of allele $A_{i}$ in newborns and the number of newborns at time $t$.

\section{Changes in Allele Frequencies}

Under weak selection and with a stable stage distribution, the change in allele frequency in stage 1 individuals can be written as

$$
\Delta p_{i}(1, t)=p_{i}(1, t)\left(\frac{\lambda_{i}}{\bar{\lambda}}-1\right)+O\left(\epsilon^{2}\right),
$$

(see the appendix for derivation). Therefore, in order for $\Delta p_{i}>0$, we need $\lambda_{i}>$ $\bar{\lambda}$, where $\lambda_{i}=\Sigma_{j} p_{j} \lambda_{i j}$ and

$$
\bar{\lambda} \approx \sum_{i} \sum_{j} p_{i} p_{j} \lambda_{i j}+O\left(\epsilon^{2}\right),
$$

where $\lambda_{i j}$ is the geometric rate of increase for a population of $A_{i} A_{j}$ individuals at the stable stage distribution. For a locus with two alleles, $A_{1}$ and $A_{2}$, allele $A_{2}$ will increase in frequency if $\lambda_{2}>\bar{\lambda}$ or

$$
p_{1} \lambda_{12}+p_{2} \lambda_{22}>p_{1}\left(p_{1} \lambda_{11}+p_{2} \lambda_{12}\right)+p_{2}\left(p_{1} \lambda_{12}+p_{2} \lambda_{22}\right) \text {. }
$$

Rearranging this gives

$$
p_{1}\left(\lambda_{12}-\lambda_{11}\right)+p_{2}\left(\lambda_{22}-\lambda_{12}\right)>0
$$

\section{Senescence}

We would like to consider a "general"' stage-classified clonal life history, that is, a life history that takes into account the major differences between clonal and aclonal organisms. Obviously, given the great variety of types of clonal reproduction and of organisms exhibiting such reproduction, any one model will miss many important details. However, two important features of clonal organisms deserve special attention. First, the production of genetically identical "pieces" of the genetic individual, or genet, results in circumstances not encountered by aclonal organisms. The physiological individual, or ramet, and the genet need not be identical; each ramet, although sharing the same genotype, may function and survive, or not survive, on its own. This is true whether the ramet is made up of a single module, as is the case in hydra and sea anemones, or many connected modules, such as in some grasses and corals. Second, the growth and development of many clonal organisms is often only weakly correlated with age, as 


$\left(\begin{array}{cccccccc}\mathrm{P}_{1} & \mathrm{~F}_{2} & \mathrm{~F}_{3} & \cdots & \mathrm{F}_{z} & \cdots & \cdots & \mathrm{F}_{n} \\ \mathrm{G}_{1} & \mathrm{P}_{2} & 0 & 0 & 0 & 0 & 0 & 0 \\ 0 & \mathrm{G}_{2} & \mathrm{P}_{3} & 0 & 0 & 0 & 0 & 0 \\ 0 & 0 & \mathrm{G}_{3} & \ddots & 0 & 0 & 0 & 0 \\ 0 & 0 & 0 & \ddots & \mathrm{P}_{z} & \mathrm{C}_{z+1} & \cdots & \mathrm{C}_{n} \\ 0 & 0 & 0 & 0 & \mathrm{G}_{z} & \mathrm{P}_{z+1} & 0 & 0 \\ 0 & 0 & 0 & 0 & 0 & \ddots & \ddots & 0 \\ 0 & 0 & 0 & 0 & 0 & 0 & \mathrm{G}_{n-1} & \mathrm{P}_{n}\end{array}\right)$

Fig. 1.-Transition matrix for a stage-classified clonal life history. The $i, j$ th entry gives the number of stage $i$ individuals produced per stage $j$ individual. $P_{i}$, Survival rate of stage $i$ individuals; $G_{i}$, growth rate from stage $i$ to stage $i+1 ; F_{i}$, fecundity or number of sexual offspring per stage $i$ individual; $C_{i}$, number of clonal offspring per stage $i$ individual. Stage 1 , newborn or product of sexual reproduction; stage $z$, product of clonal reproduction.

measured by time since zygote formation. Thus, it is movement between particular stages that interests us on a demographic level.

We consider an organism with stages 1 through $n$ and two types of reproduction: sexual reproduction, which produces stage 1 individuals; and clonal reproduction, which in the simplest case produces individuals of one stage, $z$. The transition matrix $\mathbf{A}$ has the general form given in figure 1: the $P_{i}$ 's give survival rates, which are probabilities of remaining in the same stage; the $G_{i}$ 's give "growth" rates, or rates of movement to the next stage; the $F_{i}$ 's govern production of sexual offspring; and the $C_{i}$ 's give production of clonal offspring. This allows the two main features, production of new physiological individuals with the same genotype and indeterminate growth, to be addressed.

Consider an allele, $A_{2}$, with pleiotropic effects on two sets of life-history stages, $S_{1}$ and $S_{2}$. In order to consider senescence, we may wish to assume that this allele increases $P_{x}$ for stages $x \in S_{1}$ and decreases $P_{y}$ for stages $y \in S_{2}$. Thus, if stage $x$ individuals are on the average younger than stage $y$ individuals, this allele causes a greater decrease in survival with age and leads to senescence. Note that for this model, $P_{x}=a_{x x}$.

We first consider the case in which the effects are limited to two stages: $S_{1}=$ $\{x\}$ and $S_{2}=\{y\}$. Assuming the allele has additive effects, so that the relationships in table 1 hold, we have

$$
\begin{aligned}
& P_{x}^{\langle 1,2\rangle}-P_{x}^{\langle 1,1\rangle}=a_{x x}^{\langle 1,2\rangle}-a_{x x}^{\langle 1,1\rangle}=\alpha, \\
& P_{x}^{\langle 2,2\rangle}-P_{x}^{\langle 1,2\rangle}=a_{x x}^{\langle 2,2\rangle}-a_{x x}^{\langle 1,2\rangle}=\alpha, \\
& P_{y}^{\langle 1,2\rangle}-P_{y}^{\langle 1,1\rangle}=a_{y y}^{\langle 1,2\rangle}-a_{y y}^{\langle 1,1\rangle}=-\beta, \\
& P_{y}^{\langle 2,2\rangle}-P_{y}^{\langle 1,2\rangle}=a_{y y}^{\langle 2,2\rangle}-a_{y y}^{\langle 1,2\rangle}=-\beta,
\end{aligned}
$$


TABLE 1

Effect of Allele $A_{2}$ on Survival Rates for Life-History STAGes $x$ AND $y$

\begin{tabular}{lllc}
\hline \hline & \multicolumn{3}{c}{ Genotype } \\
\cline { 2 - 4 } SuRvival Rates & $A_{1} A_{1}$ & $A_{1} A_{2}$ & $A_{2} A_{2}$ \\
\hline$P_{x}$ & $P_{x}^{\langle 1,1\rangle}$ & $P_{x}^{\langle 1,1\rangle}+\alpha$ & $P_{x}^{\langle 1,1\rangle}+2 \alpha$ \\
$P_{y}$ & $P_{y}^{\langle 1,1\rangle}$ & $P_{y}^{\langle 1,1\rangle}-\beta$ & $P_{y}^{\langle 1,1\rangle}-2 \beta$ \\
\hline
\end{tabular}

where the remaining $a_{k l}$ are the same for all genotypes. Then, using equations (A9) and (A10) from the appendix,

$$
\left(\lambda_{12}-\lambda_{11}\right)=\alpha \frac{\partial \lambda_{11}}{\partial a_{x x}^{\langle 1,1\rangle}}-\beta \frac{\partial \lambda_{11}}{\partial a_{y y}^{\langle 1,1\rangle}},
$$

and

$$
\left(\lambda_{22}-\lambda_{12}\right)=\alpha \frac{\partial \lambda_{12}}{\partial a_{x x}^{\langle 1,2\rangle}}-\beta \frac{\partial \lambda_{12}}{\partial a_{y y}^{\langle 1,2\rangle}} .
$$

In order for $\Delta p_{2}>0$,

$$
p_{1}\left(\alpha \frac{\partial \lambda_{11}}{\partial a_{x x}^{\langle 1,1\rangle}}-\beta \frac{\partial \lambda_{11}}{\partial a_{y y}^{\langle 1,1\rangle}}\right)+p_{2}\left(\alpha \frac{\partial \lambda_{12}}{\partial a_{x x}^{\langle 1,2\rangle}}-\beta \frac{\partial \lambda_{12}}{\partial a_{y y}^{\langle 1,2\rangle}}\right)>0 .
$$

In the same way, we can consider trade-offs between late survival and early reproduction, either sexual or clonal, and between sexual and clonal reproduction. For example, if the allele increases sexual reproduction at stage $x$ while decreasing survival at stage $y$, the appropriate condition for the increase of the $A_{2}$ allele is

$$
p_{1}\left(\alpha \frac{\partial \lambda_{11}}{\partial a_{1 x}^{\langle 1,1\rangle}}-\beta \frac{\partial \lambda_{11}}{\partial a_{y y}^{\langle 1,1\rangle}}\right)+p_{2}\left(\alpha \frac{\partial \lambda_{12}}{\partial a_{1 x}^{\langle 1,2\rangle}}-\beta \frac{\partial \lambda_{12}}{\partial a_{y y}^{\langle 1,2\rangle}}\right)>0
$$

We can also consider two sets of stages, $S_{1}$ and $S_{2}$, in which allele $A_{2}$ increases some entry of the transition matrix for stages in $S_{1}$ and decreases the same or a different entry for stages in $S_{2}$. For example, consider a locus in which

$$
\begin{aligned}
& F_{x}^{\langle 1,2\rangle}-F_{x}^{\langle 1,1\rangle}=a_{1 x}^{\langle 1,2\rangle}-a_{1 x}^{\langle 1,1\rangle}=\alpha_{x}, \\
& F_{x}^{\langle 2,2\rangle}-F_{x}^{\langle 1,2\rangle}=a_{1 x}^{\langle 2,2\rangle}-a_{1 x}^{\langle 1,2\rangle}=\alpha_{x}, \\
& P_{y}^{\langle 1,2\rangle}-P_{y}^{\langle 1,1\rangle}=a_{y y}^{\langle 1,2\rangle}-a_{y y}^{\langle 1,1\rangle}=-\beta_{y}, \\
& P_{y}^{\langle 2,2\rangle}-P_{y}^{\langle 1,2\rangle}=a_{y y}^{\langle 2,2\rangle}-a_{y y}^{\langle 1,2\rangle}=-\beta_{y},
\end{aligned}
$$


TABLE 2

Effect of Allele $A_{2}$ on Fecundity and Survival for Sets of

Life-History Stages $S_{1}$ and $S_{2}$, Where $x \in S_{1}$ ANd $y \in S_{2}$

\begin{tabular}{llll}
\hline \hline Life-History & \multicolumn{3}{c}{ GenOTYPE } \\
\cline { 2 - 4 } PARAMETERS & $A_{1} A_{1}$ & $A_{1} A_{2}$ & $A_{2} A_{2}$ \\
\hline$F_{x}$ & $F_{x}^{\langle 1,1\rangle}$ & $F_{x}^{\langle 1,1\rangle}+\alpha_{x}$ & $F_{x}^{\langle 1,1\rangle}+2 \alpha_{x}$ \\
$\boldsymbol{P}_{y}$ & $\boldsymbol{P}_{y}^{\langle 1,1\rangle}$ & $\boldsymbol{P}_{y}^{\langle 1,1\rangle}-\beta_{y}$ & $\boldsymbol{P}_{y}^{\langle 1,1\rangle}-2 \beta_{y}$ \\
\hline
\end{tabular}

for $x \in S_{1}, y \in S_{2}$, and in which, for all $k, l \notin S_{1}, S_{2}$, the $a_{k l}$ are the same for all genotypes (see table 2). Then we have

$$
\begin{gathered}
p_{1}\left(\sum_{x \in S_{1}} \alpha_{x} \frac{\partial \lambda_{11}}{\partial a_{1 x}^{\langle 1,1\rangle}}-\sum_{y \in S_{2}} \beta_{y} \frac{\partial \lambda_{11}}{\partial a_{y y}^{\langle 1,1\rangle}}\right) \\
+p_{2}\left(\sum_{x \in S_{1}} \alpha_{x} \frac{\partial \lambda_{12}}{\partial a_{1 x}^{\langle 1,2\rangle}}-\sum_{y \in S_{2}} \beta_{y} \frac{\partial \lambda_{12}}{\partial a_{y y}^{\langle 1,2\rangle}}\right)>0
\end{gathered}
$$

as the condition for the increase in frequency of allele $A_{2}$.

\section{NUMERICAL EXAMPLES}

To investigate the role of clonal life histories on the spread of an allele altering life-history parameters, we consider demographic data adapted from Babcock (1991). Babcock gives size-specific transition matrices for three species of scleractinian corals: Goniastrea aspera, Goniastrea favulus, and Platygyra sinensis. The matrices give transitions for five life-history stages: stage 1 (larvae, prior to visible recruitment), stage 2 (visible to $40 \mathrm{~cm}^{2}$ ), stage 3 (greater than $40 \mathrm{~cm}^{2}$ through $80 \mathrm{~cm}^{2}$ ), stage 4 (greater than $80 \mathrm{~cm}^{2}$ through $120 \mathrm{~cm}^{2}$ ), and stage 5 (greater than $120 \mathrm{~cm}^{2}$ ).

Calculating the eigenvalue sensitivities is computationally intensive; therefore, these five stages were collapsed into four by combining stages 4 and 5 into a new stage $4^{\prime}$. Entries for movement into and out of stage $4^{\prime}$ were averages of those for stages 4 and 5 , weighted so that the total movement into and out of stage $4^{\prime}$ equaled that of stages 4 and 5 combined. Movement between these stages is as in figure 2, and the adapted transition matrices are given in table 3 . The matrices as given by Babcock (1991) do not include entries for the probability of remaining within the larval stage. This is the probability of remaining in this stage for $1 \mathrm{yr}$; a zero probability was assigned for the purposes of this example.

\section{Early versus Late Survival}

We first consider an allele that has pleiotropic effects on two life-history stages, stages 2 and $4^{\prime}$, increasing survival in stage $2\left(P_{2}\right.$ or $\left.a_{22}\right)$ by an amount $\alpha$ while decreasing survival in stage $4^{\prime}\left(P_{4^{\prime}}\right.$ or $\left.a_{4^{\prime} 4^{\prime}}\right)$ by the same amount $\alpha$. The parameter 


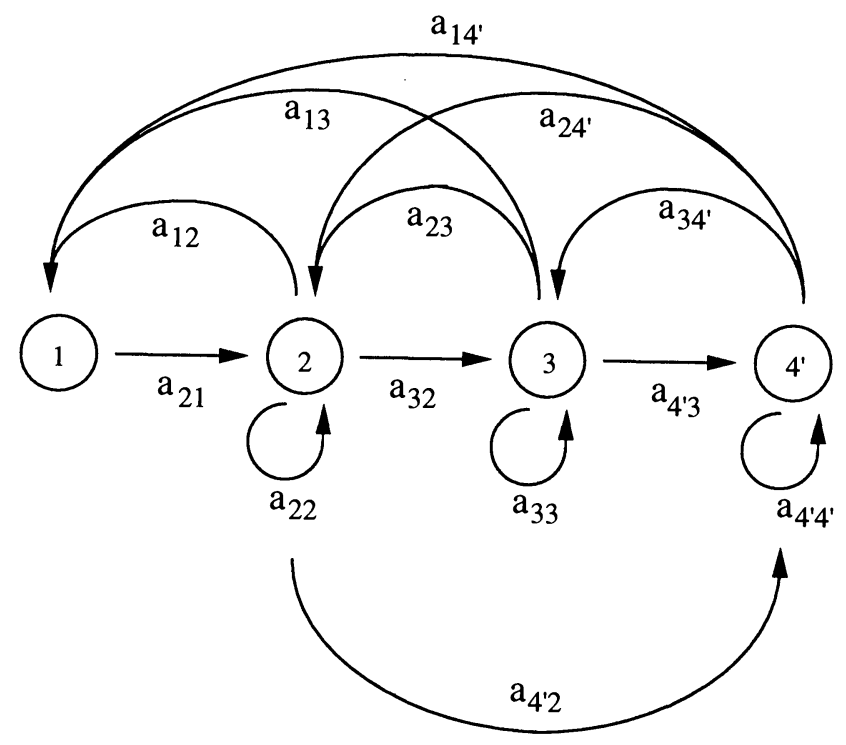

Fig. 2. - Life-cycle graph for coral demographic data adapted from Babcock (1991). The number of individuals in stage $i$ at time $t+1$ per individual in stage $j$ at time $t$ is given by the coefficient $a_{i j}$, which are the entries of the stage-classified transition matrices. Stage 1, larvae; stages 2, 3, and 4', size classes of coral colonies. Note that stages 4 and 5 in Babcock (1991) have been combined to form stage $4^{\prime}$.

TABLE 3

Transition Matrices Adapted from Babcock (i99I)

\begin{tabular}{|c|c|c|c|c|}
\hline \multirow[t]{2}{*}{ Species } & \multicolumn{4}{|c|}{ Matrix } \\
\hline & 0 & 1900 & 27000 & 183890 \\
\hline \multirow[t]{4}{*}{ Goniastrea aspera } & $1.9 \times 10^{-6}$ & .58 & .21 & .0464 \\
\hline & 0 & .1 & .53 & .0649 \\
\hline & 0 & 0 & .14 & .8309 \\
\hline & 0 & 560 & 14000 & 203751 \\
\hline \multirow[t]{4}{*}{ Goniastrea favulus } & $2.2 \times 10^{-5}$ & .77 & .28 & .1227 \\
\hline & 0 & .04 & .49 & .1454 \\
\hline & 0 & 0 & .13 & .6639 \\
\hline & 0 & 48 & 4500 & 168995 \\
\hline \multirow[t]{3}{*}{ Platygyra sinensis } & $1.9 \times 10^{-6}$ & .34 & .19 & .0338 \\
\hline & 0 & .17 & .48 & .0572 \\
\hline & 0 & .02 & .1 & .8691 \\
\hline
\end{tabular}


TABLE 4

Change in Allele Frequency for an Allele That InCREASES EARly Survival at THE Cost of Late Survival

\begin{tabular}{lc}
\hline \hline Species & $\begin{array}{c}\text { Change in } \\
\text { Allele Frequency }\end{array}$ \\
\hline Goniastrea aspera & $\Delta p_{2}<0$ \\
Goniastrea favulus & $\Delta p_{2}>0$ \\
Platygyra sinensis & $\Delta p_{2}<0$ \\
\hline
\end{tabular}

NotE.-For all three species, parameter ranges investigated were $0 \leq p_{2} \leq 1,0 \leq \alpha \leq 0.05$.

$\alpha$ must be relatively small so that the change in allele frequency per unit time is small and our assumption of weak selection holds. The condition for increase of this new allele, $A_{2}$, is given by equation (5) in which $\beta=\alpha$ and $x=2, y=4^{\prime}$. This condition was calculated for all three coral species, for $p_{2}$ (frequency of $A_{2}$ ) between zero and one and for $0 \leq \alpha \leq 0.05$. The results are given in table 4 . For two species, $G$. aspera and $P$. sinensis, the $A_{2}$ allele will not increase for any of the parameter values investigated, which indicates that senescence caused by alleles with negative pleiotropic effects on survival for the stages observed in this study will not evolve in these species. For G. favulus, however, the change in allele frequency is nonnegative throughout the parameter space; here, increases in early survival will more than offset decreases in late survival, which will allow senescence to evolve.

\section{Early Reproduction versus Late Survival}

In order to evaluate the fate of an allele increasing early reproduction at a cost of decreased late survival, we consider an allele with the following effect: an increase in sexual reproduction of an amount $\alpha$ in stages 2 and 3 and a decrease in survival of amount $\beta$ in stages 3 and $4^{\prime}$. This means that the effect of allele $A_{2}$ is as in table 2, where $S_{1}=\{2,3\}, S_{2}=\left\{3,4^{\prime}\right\}, \alpha_{x}=\alpha$ for all $x$ and $\beta_{y}=\beta$ for all $y$.

We consider initial frequencies of $A_{2}$ from zero to one, $0 \leq \beta \leq 0.05$, and $10^{3} \beta$ $\leq \alpha \leq 10^{8} \beta$ (G. aspera), $10^{3} \beta \leq \alpha \leq 10^{6} \beta$ (G. favulus), $10^{3} \beta \leq \alpha \leq 10^{7} \beta$ (P. sinensis). The results are given in table 5 , where the ratio of $\alpha / \beta$ at which the change in allele frequency becomes positive indicates the amount of increased early reproduction necessary to compensate for the decreased late survival. For both $G$. aspera and $P$. sinensis, $\alpha / \beta$ must be at least $10^{6}$, which indicates that, for $\beta$ near 0.01 , the increase in fertility would have to be nearly 10,000 offspring. This translates into an increase of over 500\% and 37\% in stages 2 and 3 for $G$. aspera; for $P$. sinensis, fertility would need to be over $20,000 \%$ and $200 \%$ that currently seen for stages 2 and 3. For G. favulus, an $\alpha / \beta$ of $5 \times 10^{4}$ gives positive changes in allele frequency. For $\beta$ near 0.01 , the corresponding increase in fertility is 500 offspring. This corresponds to an increase in fertility of $89 \%$ and $3.6 \%$ in stages 2 and 3 , respectively. 
TABLE 5

Change in Allele Frequency for an Allele That InCREASES EARLy REPRODUCTION AT THE Cost of Late Survival

\begin{tabular}{lc}
\hline \hline Change in Allele Frequency & Ratio $\alpha / \beta$ \\
\hline Goniastrea aspera: & \\
$\Delta p_{2}<0$ & $7.5 \times 10^{5}$ \\
$\Delta p_{2} \geq 0$ & $10^{6}$ \\
Goniastrea favulus: & $2.5 \times 10^{4}$ \\
$\Delta p_{2}<0$ & $5 \times 10^{4}$ \\
$\Delta p_{2}>0$ & $10^{6}$ \\
Platygyra sinensis: & $5 \times 10^{6}$ \\
$\Delta p_{2}<0$ & \\
$\Delta p_{2} \geq 0$ &
\end{tabular}

NoTE.-For all three species, parameter ranges investigated were $0 \leq p_{2} \leq 1,0 \leq \beta \leq 0.05$. Ratio $\alpha / \beta$ at which the change in allele frequency becomes positive indicates the amount of increased early sexual reproduction $(\alpha)$ necessary to compensate for the decrease in late survival ( $\beta)$.

These parameter values, with the possible exception of the change in stage 3 reproduction for $G$. favulus, violate the assumption of small changes in the transition matrix and weak selection. This indicates that, for small relative increases in early reproduction at the cost of small decreases in late survival, alleles causing the evolution of senescence will not spread.

\section{Effects of Decreased and Increased Clonal Reproduction}

For $G$. favulus, the effect of clonal reproduction on the conditions for the spread of an allele increasing early reproduction at the cost of late survival was investigated. Parameters were as in the previous section, and two cases were considered: no clonal reproduction, where the $a_{23}, a_{24}$, and $a_{34}$ entries of the transition matrix were set to zero; and increased clonal reproduction, where the $a_{23}, a_{24}$, and $a_{34}$ entries of the transition matrix were doubled. The ratio of $\alpha / \beta$ that allowed for increase in the $A_{2}$ allele was in all cases close to that of the unchanged matrix. However, in all cases the change in frequency of the $A_{2}$ allele was more positive for the case of no clonal reproduction than for the case of increased clonal reproduction, with the change in frequency of the $A_{2}$ allele for the unchanged matrix falling in between. Graphs of the change in allele frequency as a function of the initial allele frequency for both cases are given for two sets of parameters $\left(\beta=0.05, \alpha / \beta=10^{4}\right.$ and $\left.\beta=0.05, \alpha / \beta=5 \times 10^{4}\right)$ in figure 3 . This result has the intuitive explanation that clonal reproduction, by increasing the total productivity of late stages, decreases the importance of early sexual reproduction and increases the strength of selection on later stages.

\section{DISCUSSION}

The model developed in this article allows the analysis of allele frequency changes for genes affecting life-history parameters in organisms with complex life 
(A)

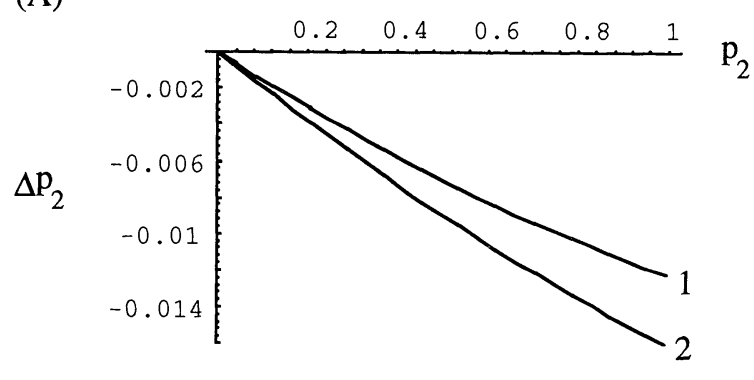

$$
\begin{aligned}
& \alpha / \beta=10^{4} \\
& \beta=0.05
\end{aligned}
$$

(B)

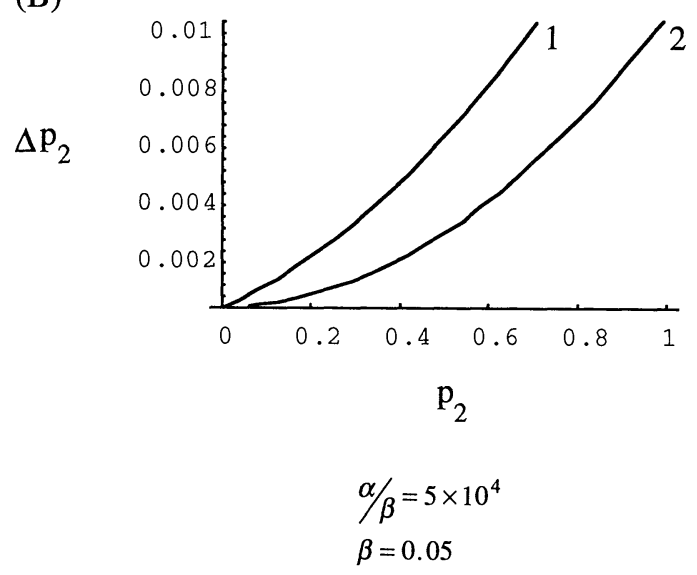

FIG. 3.-Change in allele frequency $\left(\Delta p_{2}\right)$ as a function of initial allele frequency $\left(p_{2}\right)$ for an allele increasing early reproduction at the cost of last survival for Goniastrea favulus. Curve 1, no clonal reproduction; curve 2, doubled clonal reproduction. Parameters are $(A)$ $\beta=0.05, \alpha / \beta=10^{4} ;(B) \beta=0.05, \alpha / \beta=5 \times 10^{4} 5$. See text for details.

histories. It allows conditions to be defined for the increase of alleles favoring the evolution of senescence in such organisms, in a way analogous to the approach for age-structured organisms (Charlesworth 1980), under assumptions of random mating and density and frequency independence. Whether alleles decreasing late fitness components while increasing early fitness components will spread in a population will depend on the particular demographics of that population. The sensitivity of the geometric rate of increase of the population to particular demographic parameters will not be a simple function for organisms with indeterminate growth and multiple modes of reproduction, and it is these sensitivities that determine the spread of alleles allowing the evolution of senescence. 
The biological examples examined here give an idea of the effects of these complications. These examples allow further exploration of the analytical results, but they do suffer from the problem of not considering the possible evolution of decreased fitness on stage or age classes not observed in the demographic studies. Only by using demographic data from laboratory studies in which the researcher is confident of having observed the "maximum" age or all possible stages could this problem be overcome. In determining the fate of alleles that decrease late survival rate while increasing early survival rate or reproduction, it was most often found that such alleles would not spread, given the parameter space allowed by the assumptions of the model. However, this was not always true, and in one case (Goniastrea favulus; table 4) the opposite trend was found. Whether senescence evolves would seem to depend strongly on the particular biological details of the population's demographic parameters, although the types of life histories examined here would seem to be good candidates for nonsenescing species, since these corals show indeterminate growth, clonal reproduction, and increasing fecundity with size. Babcock (1991) was able to use size measurements as well as growth rate data from X-rays of adult corals to estimate ages. From this data, he concluded that there is no evidence for physiological senescence in these corals, although limits to potential life span do arise from morphological and habitat considerations.

Certainly there is data that imply that some organisms may escape the evolution of senescence. Cook (1983) lists life spans of some clonal organisms that range from over $330 \mathrm{yr}$ to over $13,000 \mathrm{yr}$, although long life span in and of itself is not proof of the lack of senescence (see Finch 1990, chap. 4). Hughes and Jackson (1985) found no evidence of senescence in a study of five species of foliaceous corals; however, Palumbi and Jackson (1983) found evidence of zooid senescence in Steginoporella, a clonal bryozoan, although what this means at the level of individual senescence is unclear, since in this species zooids are not physiologically independent individuals. Bell (1984) gives results showing that two asexual oligochaetes (Aelosoma tenebrarum and Pristina aequiseta) do not have decreasing survival rates with age. There is also data on hydra and flatworms to this effect (see Bell 1988). However, a recent study of another asexual marine oligochaete, Paranais litoralis, by Martínez and Levinton (1992) does show decreasing survival with age. It seems that predicting where senescence will occur may not be a simple matter of deciding whether germline and soma are distinct but will depend on the biological details of the life history of the organisms. Given these details, in the form of life-history parameters, the model presented in this article predicts when the strength of selection on alleles with stage-specific effects will allow senescence to evolve.

Clonal reproduction was found in this analysis to retard the evolution of senescence, although in and of itself, the existence of clonal reproduction in an organism does not preclude senescence. Changes in the amount of clonal reproduction affected the magnitude of the change in allele frequency but not the sign. This means that the amount of clonal reproduction will affect the rate of spread of alleles causing senescence and thus the relative strength of selection for or against these alleles in the face of genetic drift.

In addition to the action of alleles having pleiotropic fitness effects, another 
major theory for how the weakening of the force of selection with age or stage allows senescence to evolve is the accumulation of deleterious mutations affecting specific stages. Stage-specific deleterious mutations would move to frequencies determined by a balance between drift due to finite population size and selection against them. In order for senescence to evolve by this mechanism, the strength of selection against alleles affecting later stages would have to be less than the strength of selection against alleles affecting earlier stages. The change in allele frequency due to selection for an allele affecting one or a set of life-history parameters negatively, without increasing any life-history parameters, would still be given by equation (3). Antagonistic pleiotropy gives an allele causing decreased survival or fecundity a better chance to increase in frequency; alleles without positive effects to offset deleterious effects have a lesser probability of eventually fixing, although the accumulation of low-frequency mutations could also be important. Further work needs to be done to explore the effects of the accumulation of deleterious mutations in age- and stage-structured populations. A promising avenue of investigation would be to extend the model of Partridge and Barton (1993).

It is important to note that in the type of clonal reproduction analyzed here, there is production of an individual of a different stage than the "parent." In organisms that produce offspring identical to themselves, there are no stage- or age-specific differences in the life history and thus no change in the force of selection, and senescence would not be expected to evolve.

The model and methods developed in this article allow demographic data describing transitions between life-history stages to be used in examining the strength of selection on alleles changing those demographic parameters. This kind of data, although difficult to gather, has been collected for many plants and marine invertebrates in which clonal reproduction and complex life histories are found (see, e.g., Werner and Caswell 1977; Bullock 1980; Hughes 1984; Hughes and Jackson 1985; Lasker 1990; Babcock 1991; McFadden 1991). It is possible, as the results of this article and others show, to extend the concepts of population genetics that were originally established for organisms with relatively simple life histories to those that include clonal reproduction. For example, Cochran and Ellner (1992) give methods for calculating standard age-based life-history measures, such as survival rate functions, maternity functions, and net reproductive rate, from stage-structured matrix models. A method for determining the inbreeding effective population size for stage-structured models is described elsewhere (Orive 1993). The resulting models and predictions, although regrettably more complex, will allow the investigation of life-history evolution in ecologically interesting organisms, such as many clonal plants and marine invertebrates, using the tools of evolutionary theory.

\section{ACKNOWLEDGMENTS}

I would like to thank M. Slatkin for guidance and suggestions throughout this work; S. Otto, L. Partridge, and T. Robinson for helpful discussion; and B. Charlesworth for clarification of a theoretical point from his text. The helpful comments of two anonymous reviewers are gratefully acknowledged. This re- 
search has been supported by a National Science Foundation Graduate Fellowship to me and grant GM40282 from the National Institutes of Health to M. Slatkin.

\section{APPENDIX \\ Derivation of Allele Frequency Equations and Conditions for InCREASE In Allele FreQuencies \\ EQUILIBRIUM ALLELE FREQUENCIES}

At genetic equilibrium, $p_{i}(x, t)=\hat{p}_{i}(x)$. Using equation $\left(2 \mathrm{a}^{\prime}\right)$ and rearranging terms gives

$$
1=\sum_{j} \hat{p}_{j}(1) \sum_{x} a_{1 x}^{\langle i, j\rangle} \sum_{\tau=1}^{\infty} \frac{B[1, t-(\tau+1)]}{B(1, t)}\left[\left(\tilde{a}^{\langle i, j\rangle}\right)_{x 1}^{\tau}-\left(\tilde{a}^{\langle i, j\rangle}\right)_{x 1}^{\tau-1}\right] .
$$

At some time $t^{\prime}$, the population will reach demographic equilibrium, and each stage class will grow at the same rate as the whole population. We can then write

$$
\frac{B\left(1, t^{\prime}-x\right)}{B\left(1, t^{\prime}\right)} \approx \bar{\lambda}^{-x}
$$

where $\bar{\lambda}$ is the leading nonunit eigenvalue of the $\mathbf{A}$ matrix for a population at the equilibrium gene frequencies. This value, $\bar{\lambda}$, is the geometric rate of increase of the population. Then, equation (A1) becomes, approximately,

$$
1=\sum_{j} \hat{p}_{j}(1) \sum_{x} a_{1 x}^{\langle i, j\rangle} \sum_{\tau=1}^{\infty} \bar{\lambda}^{-(\tau+1)}\left[\left(\tilde{a}^{\langle i, j\rangle}\right)_{x 1}^{\tau}-\left(\tilde{a}^{\langle i, j\rangle}\right)_{x 1}^{\tau-1}\right]
$$

If we define the mean fitness of a population at equilibrium as $\bar{w}=\Sigma_{i} \Sigma_{j} \hat{p}_{i}(1) \hat{p}_{j}(1) w_{i j}$ and $1=\Sigma_{j} \hat{p}_{j}(1)\left(w_{i j} / \bar{w}\right)$, letting $\bar{w}=1$, we can then define

$$
w_{i j}(\bar{\lambda})=\sum_{x} a_{1 x}^{\langle i, j\rangle} \sum_{\tau} \bar{\lambda}^{-(\tau+1)}\left[\left(\tilde{a}^{\langle i, j\rangle}\right)_{x 1}^{\tau}-\left(\tilde{a}^{\langle i, j\rangle}\right)_{x 1}^{\tau-1}\right],
$$

keeping in mind that all summations over $\tau$ are for $\tau=1$ to $\infty$. This equilibrium fitness is analogous to the equilibrium fitness function for age-structured populations; it is the contribution of the $A_{i} A_{j}$ genotype at the newborn stage to the reproductive value of the whole population (see Charlesworth 1980, sec. 3.3). The equilibrium allele frequencies for stages other than newborns depend on the $\hat{p}_{i}(1)$ and can be written as

$$
\hat{p}_{i}(x)=\hat{p}_{i}(1) \frac{\sum_{j} \hat{p}_{j}(1) \sum_{\tau} B(1, t-\tau)\left[\left(\tilde{a}^{\langle i, j\rangle}\right)_{x 1}^{\tau}-\left(\tilde{a}^{\langle i, j\rangle}\right)_{x 1}^{\tau-1}\right]}{\sum_{i} \sum_{j} \sum_{x} \hat{p}_{i}(1) \hat{p}_{j}(1) \sum_{\tau} B(1, t-\tau)\left[\left(\tilde{a}^{\langle i, j\rangle}\right)_{x 1}^{\tau}-\left(\tilde{a}^{\langle i, j\rangle}\right)_{x 1}^{\tau-1}\right]} .
$$

CHANGES IN ALLELE FREQUENCIES

In order to calculate the change in frequency of an allele $A_{i}$ in stage 1 individuals, we let

$$
\frac{B[1, t-(\tau+1)]}{B(1, t)} \approx \bar{\lambda}^{-(\tau+1)}
$$

and rewrite equation $\left(2 a^{\prime}\right)$ as

$$
p_{i}(1, t)=\sum_{j} \sum_{x} a_{1 x}^{\langle i, j\rangle} \sum_{\tau=1}^{\infty} p_{i}[1, t-(\tau+1)] p_{j}[1, t-(\tau+1)] \bar{\lambda}^{-(\tau+1)}\left[\left(\tilde{a}^{\langle i, j\rangle}\right)_{x 1}^{\tau}-\left(\tilde{a}^{\langle i, j\rangle}\right)_{x 1}^{\tau-1}\right] .
$$


If we assume that, under weak selection and with a stable stage distribution, the rate of change of allele frequency is approximately constant over several time units, we can let $p_{i}(1, t-x)=p_{i}(1, t)-x \Delta p_{i}(1, t)$ (Haldane 1927). Assuming $\Delta p_{i} \approx O(\epsilon)$ and $\Delta^{2} p_{i} \approx O\left(\epsilon^{2}\right)$ and that terms of $O\left(\epsilon^{2}\right)$ can be neglected compared to terms of $O(\epsilon)$, this gives

$$
\begin{aligned}
p_{i}(1, t)= & \sum_{j} \sum_{x} a_{1 x}^{\langle i, j\rangle} \sum_{\tau}\left[p_{i}(1, t)-(\tau+1) \Delta p_{i}(1, t)\right] \\
& \times\left[p_{j}(1, t)-(\tau+1) \Delta p_{j}(1, t)\right] \bar{\lambda}^{-(\tau+1)}\left[\left(\tilde{a}^{\langle i, j\rangle}\right)_{x 1}^{\tau}-\left(\tilde{a}^{\langle i, j\rangle}\right)_{x 1}^{\tau-1}\right] \\
= & p_{i}(1, t) \sum_{j} p_{j}(1, t) \sum_{x} a_{1 x}^{\langle i, j\rangle} \sum_{\tau} \bar{\lambda}^{-(\tau+1)}\left[\left(\tilde{a}^{(i, j\rangle}\right)_{x 1}^{\tau}-\left(\tilde{a}^{\langle i, j\rangle}\right)_{x 1}^{\tau-1}\right] \\
& -p_{i}(1, t) \sum_{j} \Delta p_{j}(1, t) \sum_{x} a_{1 x}^{\langle i, j\rangle} \sum_{\tau}(\tau+1) \bar{\lambda}^{-(\tau+1)}\left[\left(\tilde{a}^{\langle i, j\rangle}\right)_{x 1}^{\tau}-\left(\tilde{a}^{\langle i, j\rangle}\right)_{x 1}^{\tau-1}\right] \\
& -\Delta p_{i}(1, t) \sum_{j} p_{j}(1, t) \sum_{x} a_{1 x}^{\langle i, j\rangle} \sum_{\tau}(\tau+1) \bar{\lambda}^{-(\tau+1)}\left[\left(\tilde{a}^{\langle i, j\rangle}\right)_{x 1}^{\tau}-\left(\tilde{a}^{\langle i, j\rangle}\right)_{x 1}^{\tau-1}\right]+O\left(\epsilon^{2}\right) .
\end{aligned}
$$

Concentrating on the second term in this equation, we note that $\Sigma_{j} \Delta p_{j}(1, t)=0$, and we let

$$
a_{1 x}^{\langle i, j\rangle}\left[\left(\tilde{a}^{\langle i, j\rangle}\right)_{x 1}^{\tau}-\left(\tilde{a}^{\langle i, j\rangle}\right)_{x 1}^{\tau-1}\right] \approx a_{1 x}\left[(\tilde{a})_{x 1}^{\tau}-(\tilde{a})_{x 1}^{\tau-1}\right]+O(\epsilon) .
$$

Here, we are assuming that the differences between the entries of the different transition matrices for any genotype are of order $\epsilon$. The second term can then be written as

$$
\begin{aligned}
p_{i}(1, t) \sum_{x} \sum_{\tau}(\tau+1) \bar{\lambda}^{-(\tau+1)} \sum_{j} \Delta p_{j}(1, t)\left\{a_{1 x}\left[(\tilde{a})_{x 1}^{\tau}-(\tilde{a})_{x 1}^{\tau-1}\right]+O(\epsilon)\right\} \\
=p_{i}(1, t) \sum_{x} \sum_{\tau}(\tau+1) \bar{\lambda}^{-(\tau+1)} a_{1 x}\left[(\tilde{a})_{x 1}^{\tau}-(\tilde{a})_{x 1}^{\tau-1}\right] \sum_{j} \Delta p_{j}(1, t) \\
+p_{i}(1, t) \sum_{x} \sum_{\tau}(\tau+1) \bar{\lambda}^{-(\tau+1)} \sum_{j} \Delta p_{j}(1, t) O(\epsilon),
\end{aligned}
$$

and, since $\Delta p_{i} \approx O(\epsilon)$, this equals $0+O\left(\epsilon^{2}\right)$. Returning to the original equation for $p_{i}(1, t)$, we have

$$
\begin{aligned}
p_{i}(1, t)= & p_{i}(1, t) \sum_{j} p_{j}(1, t) \sum_{x} a_{1 x}^{\langle i, j\rangle} \sum_{\tau} \bar{\lambda}^{-(\tau+1)}\left[\left(\tilde{a}^{\langle i, j\rangle}\right)_{x 1}^{\tau}-\left(\tilde{a}^{\langle i, j\rangle}\right)_{x 1}^{\tau-1}\right] \\
& -\Delta p_{i}(1, t) \sum_{j} p_{j}(1, t) \sum_{x} a_{1 x}^{\langle i, j\rangle} \sum_{\tau}(\tau+1) \bar{\lambda}^{-(\tau+1)}\left[\left(\tilde{a}^{\langle i, j\rangle}\right)_{x 1}^{\tau}-\left(\tilde{a}^{\langle i, j\rangle}\right)_{x 1}^{\tau-1}\right]+O\left(\epsilon^{2}\right) .
\end{aligned}
$$

Rearranging this gives

$$
\Delta p_{i}(1, t) S_{i}(\bar{\lambda})=p_{i}(1, t)\left[\sum_{j} p_{j}(1, t) w_{i j}(\bar{\lambda})-1\right]+O\left(\epsilon^{2}\right),
$$

where we define $S_{i}(\bar{\lambda})$ as

$$
S_{i}(\bar{\lambda})=\sum_{j} p_{j}(1, t) \sum_{x} a_{1 x}^{\langle i, j\rangle} \sum_{\tau}(\tau+1) \bar{\lambda}^{-(\tau+1)}\left[\left(\tilde{a}^{\langle i, j\rangle}\right)_{x 1}^{\tau}-\left(\tilde{a}^{\langle i, j\rangle}\right)_{x 1}^{\tau-1}\right],
$$

and equation (A2) gives $w_{i j}(\bar{\lambda})$. For a population of $A_{i} A_{j}$ individuals at the stable stage distribution, growing at a geometric rate $\bar{\lambda}, S_{i j}(\bar{\lambda})$ is the meiotic generation time (Orive 1993), where generation time is defined as the mean number of time units since a parent of a newborn individual was itself a newborn, and

$$
S_{i j}(\bar{\lambda})=\sum_{x} a_{1 x}^{\langle i, j\rangle} \sum_{\tau}(\tau+1) \bar{\lambda}^{-(\tau+1)}\left[\left(\tilde{a}^{\langle i, j\rangle}\right)_{x 1}^{\tau}-\left(\tilde{a}^{\langle i, j\rangle}\right)_{x 1}^{\tau-1}\right]
$$


Then, $S_{i}(\bar{\lambda})$ is a weighted generation time for a population in the stable stage distribution. Let $w_{i}(\bar{\lambda})=\Sigma_{j} p_{j}(1, t) w_{i j}(\bar{\lambda})$, and equation (A4) becomes

$$
\Delta p_{i}(1, t) S_{i}(\bar{\lambda})=p_{i}(1, t)\left[w_{i}(\bar{\lambda})-1\right]+O\left(\epsilon^{2}\right) .
$$

Equation (A7) is analogous to equation (4.9) in Charlesworth (1980) for age-structured populations. We then express the change in allele frequency as a function only of gene frequency and the genotype geometric rates of increase. We can approximate $w_{i}(\bar{\lambda})$ by using Taylor's theorem as follows:

$$
w_{i}\left(\lambda_{i}\right)=w_{i}(\bar{\lambda})+\left(\lambda_{i}-\bar{\lambda}\right)\left(\frac{\partial w_{i}(z)}{\partial z}\right)_{\bar{\lambda}}+O\left(\epsilon^{2}\right) .
$$

If we let $w_{i}\left(\lambda_{i}\right)=1$, this value becomes

$$
\begin{aligned}
w_{i}(\bar{\lambda})-1 & =-\left(\lambda_{i}-\bar{\lambda}\right)\left(\frac{\partial w_{i}(z)}{\partial z}\right)_{\bar{\lambda}}+O\left(\epsilon^{2}\right) \\
& =-\left(\lambda_{i}-\bar{\lambda}\right)\left(-\frac{1}{\bar{\lambda}}\right) S_{i}(\bar{\lambda})+O\left(\epsilon^{2}\right) \\
& =\left(\frac{\lambda_{i}}{\bar{\lambda}}-1\right) S_{i}(\bar{\lambda})+O\left(\epsilon^{2}\right) .
\end{aligned}
$$

Substituting this expression into equation (A7) gives

$$
\Delta p_{i}(1, t) S_{i}(\bar{\lambda})=p_{i}(1, t)\left[\left(\frac{\lambda_{i}}{\bar{\lambda}}-1\right) S_{i}(\bar{\lambda})\right]+O\left(\epsilon^{2}\right)
$$

or

$$
\Delta p_{i}(1, t)=p_{i}(1, t)\left(\frac{\lambda_{i}}{\bar{\lambda}}-1\right)+O\left(\epsilon^{2}\right) .
$$

EFFECTS OF STAGE-SPECIFIC GENES: ALLELES AFFECTING ONE LIFE-HISTORY PARAMETER

Initially, we consider a locus that has a stage-specific effect on one life-history parameter, $a_{x y}$. For any genotype $A_{i} A_{j}$, this parameter is $\tilde{a}_{x y}^{\langle i, j\rangle}$. For a locus with two alleles, $A_{1}$ and $A_{2}$, we need to estimate the geometric rates of increase for the possible genotypes. Imagine a population fixed for allele $A_{1}$ when allele $A_{2}$ is introduced. We can approximate the geometric rates of increase using Taylor's theorem,

$$
\begin{gathered}
\lambda_{12}=\lambda_{11}+\left(a_{x y}^{\langle 1,2\rangle}-a_{x y}^{\langle 1,1\rangle}\right)\left(\frac{\partial \lambda_{11}}{\partial a_{x y}^{\langle 1,1\rangle}}\right)+O\left(\epsilon^{2}\right), \\
\left(\lambda_{12}-\lambda_{11}\right)=\left(a_{x y}^{\langle 1,2\rangle}-a_{x y}^{\langle 1,1\rangle}\right)\left(\frac{\partial \lambda_{11}}{\partial a_{x y}^{\langle 1,1\rangle}}\right)+O\left(\epsilon^{2}\right) .
\end{gathered}
$$

Similarly,

$$
\left(\lambda_{22}-\lambda_{12}\right)=\left(a_{x y}^{\langle 2,2\rangle}-a_{x y}^{\langle 1,2\rangle}\right)\left(\frac{\partial \lambda_{12}}{\partial a_{x y}^{\langle 1,2\rangle}}\right)+O\left(\epsilon^{2}\right) .
$$

Equation (4) then becomes, approximately,

$$
p_{1}\left[\left(a_{x y}^{\langle 1,2\rangle}-a_{x y}^{\langle 1,1\rangle}\right) \frac{\partial \lambda_{11}}{\partial a_{x y}^{\langle 1,1\rangle}}\right]+p_{2}\left[\left(a_{x y}^{\langle 2,2\rangle}-a_{x y}^{\langle 1,2\rangle}\right) \frac{\partial \lambda_{12}}{\partial a_{x y}^{\langle 1,2\rangle}}\right]>0 .
$$


The entries of the sensitivity matrix for the transition matrix $\mathbf{A}^{\langle i, j\rangle}$ are $\left(\partial \lambda_{i j}\right) /\left(\partial a_{x y}^{\langle i, j\rangle}\right)$ and can be found in several ways. One method is to calculate the left and right eigenvectors $(\mathrm{v}$ and $\mathrm{w})$. From this,

$$
\frac{\partial \lambda_{i j}}{\partial a_{x y}^{\langle i, j\rangle}}=\frac{v_{x} w_{y}}{\langle\mathbf{w}, \mathbf{v}\rangle},
$$

where $\langle\mathbf{w}, \mathbf{v}\rangle$ indicates the scalar product of the eigenvectors (see Faddeev and Faddeeva 1963; Caswell 1978, 1989).

EFFECTS OF STAGE-SPECIFIC GENES: ALLELES AFFECTING A SET OF LIFE-HISTORY PARAMETERS

Again, we consider a locus with two alleles, $A_{1}$ and $A_{2}$. These alleles affect a set of life-history parameters, $S$. Equations (A9) and (A10) then become

$$
\left(\lambda_{12}-\lambda_{11}\right)=\sum_{x, y \in S}\left(a_{x y}^{\langle 1,2\rangle}-a_{x y}^{\langle 1,1\rangle}\right) \frac{\partial \lambda_{11}}{\partial a_{x y}^{\langle 1,1\rangle}}+O\left(\epsilon^{2}\right)
$$

and

$$
\left(\lambda_{22}-\lambda_{12}\right)=\sum_{x, y \in S}\left(a_{x y}^{\langle 2,2\rangle}-a_{x y}^{\langle 1,2\rangle}\right) \frac{\partial \lambda_{12}}{\partial a_{x y}^{\langle 1,2\rangle}}+O\left(\epsilon^{2}\right) .
$$

The condition for an increase in the frequency of allele $A_{2}$ becomes

$$
p_{1}\left[\sum_{x, y \in S}\left(a_{x y}^{\langle 1,2\rangle}-a_{x y}^{\langle 1,1\rangle}\right) \frac{\partial \lambda_{11}}{\partial a_{x y}^{\langle 1,1\rangle}}\right]+p_{2}\left[\sum_{x, y \in S}\left(a_{x y}^{\langle 2,2\rangle}-a_{x y}^{\langle 1,2\rangle}\right) \frac{\partial \lambda_{12}}{\partial a_{x y}^{\langle 1,2\rangle}}\right]>0 .
$$

\section{LITERATURE CITED}

Babcock, R. C. 1991. Comparative demography of three species of scleractinian corals using ageand size-dependent classifications. Ecological Monographs 61:225-244.

Bell, G. 1984. Evolutionary and nonevolutionary theories of senescence. American Naturalist 124: 600-603.

1988. Sex and death in protozoa: the history of an obsession. Cambridge University Press, Cambridge.

1992. Mid-life crisis. Evolution 46:854-856.

Bullock, S. H. 1980. Demography of an undergrowth palm in littoral Cameroon. Biotropica 12: 247-255.

Buss, L. W. 1985. The uniqueness of the individual revisited. Pages 467-505 in J. B. C. Jackson, L. W. Buss, and R. E. Cook, eds. Population biology and evolution of clonal organisms. Yale University Press, New Haven, Conn.

1987. The evolution of individuality. Princeton University Press, Princeton, N.J.

Caswell, H. 1978. A general formula for the sensitivity of population growth rate to changes in life history parameters. Theoretical Population Biology 14:215-230.

1985. The evolutionary demography of clonal reproduction. Pages 187-224 in J. B. C. Jackson, L. W. Buss, and R. E. Cook, eds. Population biology and evolution of clonal organisms. Yale University Press, New Haven, Conn.

1989. Matrix population models. Sinauer, Sunderland, Mass.

Charlesworth, B. 1970. Selection in populations with overlapping generations. I. The use of Malthusian parameters in population genetics. Theoretical Population Biology 1:352-370.

- 1974. Selection in populations with overlapping generations. VI. Rates of change of gene frequency and population growth rate. Theoretical Population Biology 6:108-132.

1980. Evolution in age-structured populations. Cambridge University Press, Cambridge.

Coates, A. G., and J. B. C. Jackson. 1985. Morphological themes in the evolution of clonal and 
aclonal marine invertebrates. Pages 67-106 in J. B. C. Jackson, L. W. Buss, and R. E. Cook, eds. Population biology and evolution of clonal organisms. Yale University Press, New Haven, Conn.

Cochran, M. E., and S. Ellner. 1992. Simple methods for calculating age-based life history parameters for stage-structured populations. Ecological Monographs 62:345-364.

Comfort, A. 1956. The biology of senescence. Holt, Rinehart \& Winston, New York.

Cook, R. E. 1983. Clonal plant populations. American Scientist 71:244-253.

Faddeev, D. K., and V. N. Faddeeva. 1963. Computational methods of linear algebra. W. H. Freeman, San Francisco.

Finch, C. E. 1990. Longevity, senescence, and the genome. University of Chicago Press, Chicago. Grosberg, R. K. 1992. Asexual obsessions. Evolution 46:1976-1979.

Haldane, J. B. S. 1927. A mathematical theory of natural and artificial selection. IV. Proceedings of the Cambridge Philosophical Society 23:607-615.

Hamilton, W. D. 1966. The moulding of senescence by natural selection. Journal of Theoretical Biology 12:12-45.

Harper, J. L. 1985. Modules, branches, and the capture of resources. Pages 1-33 in J. B. C. Jackson, L. W. Buss, and R. E. Cook, eds. Population biology and evolution of clonal organisms. Yale University Press, New Haven, Conn.

Hughes, R. N. 1989. A functional biology of clonal animals. Chapman \& Hall, London.

Hughes, T. P. 1984. Population dynamics based on individual size rather than age: a general model with a reef coral example. American Naturalist 123:778-795.

Hughes, T. P., and J. B. C. Jackson. 1985. Population dynamics and life histories of foliaceous corals. Ecological Monographs 55:141-166.

Jackson, J. B. C., L. W. Buss, and R. E. Cook, eds. 1985. Population biology and evolution of clonal organisms. Yale University Press, New Haven, Conn.

Lasker, H. R. 1990. Clonal propagation and population dynamics of a gorgonian coral. Ecology 71:1578-1589.

Lefkovitch, L. P. 1965. The study of population growth in organisms grouped by stages. Biometrics 21:1-18.

Leslie, P. H. 1945. On the use of matrices in certain population mathematics. Biometrika 33:183-212.

Martínez, D., and J. Levinton. 1992. Asexual metazoans undergo senescence. Proceedings of the National Academy of Sciences of the USA 89:9920-9923.

McFadden, C. S. 1991. A comparative demographic analysis of clonal reproduction in a temperate soft coral. Ecology 72:1849-1866.

Medawar, P. B. 1952. An unsolved problem of biology. Lewis, London.

Muller, H. J. 1964. The relation of recombination to mutational advance. Mutation Research 1:2-9.

Orive, M. E. 1993. Effective population size in organisms with complex life-histories. Theoretical Population Biology 44:316-340.

Palumbi, S. R., and J. B. C. Jackson. 1983. Aging in modular organisms: ecology of zooid senescence in Steginoporella sp. (Bryozoa: Cheilostomata). Biological Bulletin 164:267-278.

Partridge, L., and N. Barton. 1993. Optimality, mutation and the evolution of ageing. Nature (London) 362:305-311.

Rose, M. R. 1991. Evolutionary biology of aging. Oxford University Press, Oxford.

Stearns, S. C. 1992. The evolution of life histories. Oxford University Press, Oxford.

Watkinson, A. 1992. Plant senescence. Trends in Ecology \& Evolution 7:417-420.

Weismann, A. 1893. The germ-plasm: a theory of heredity. Scott, London.

1904. The evolutionary theory. Arnold, London.

Werner, P. A., and H. Caswell. 1977. Population growth rates and age versus stage-distribution models for teasel (Dipsacus sylvestris Huds.). Ecology 58:1103-1111.

Williams, G. C. 1957. Pleiotropy, natural selection, and the evolution of senescence. Evolution 11: 398-411. 